PROCEEDINGS OF THE

AMERICAN MATHEMATICAL SOCIETY

Volume 131, Number 8, Pages 2313-2323

S 0002-9939(02)06788-6

Article electronically published on November 14, 2002

\title{
VANISHING OF COHOMOLOGY OVER GORENSTEIN RINGS OF SMALL CODIMENSION
}

LIANA M. ŞEGA

(Communicated by Wolmer V. Vasconcelos)

\begin{abstract}
We prove that if $M, N$ are finite modules over a Gorenstein local ring $R$ of codimension at most 4 , then the vanishing of $\operatorname{Ext}_{R}^{n}(M, N)$ for $n \gg 0$ is equivalent to the vanishing of $\operatorname{Ext}_{R}^{n}(N, M)$ for $n \gg 0$. Furthermore, if $\widehat{R}$ has no embedded deformation, then such vanishing occurs if and only if $M$ or $N$ has finite projective dimension.
\end{abstract}

\section{INTRODUCTION}

Let $(R, \mathfrak{m}, k)$ be a commutative noetherian local ring with maximal ideal $\mathfrak{m}$ and residue field $k$. The codimension of $R$ is the number $\operatorname{codim} R=\operatorname{edim} R-\operatorname{dim} R$, where edim $R$ denotes the minimal number of generators of $\mathfrak{m}$.

Let $M, N$ be finite $R$-modules. We study the vanishing of $\operatorname{Ext}_{R}^{n}(M, N)$ for all $n \gg 0$ and the vanishing of $\operatorname{Tor}_{n}^{R}(M, N)$ for all $n \gg 0$, assuming that $R$ is Gorenstein and $M, N$ are finite $R$-modules.

It is convenient to state the vanishing properties of (co)homology in terms of numbers $e_{R}(M, N)$ and $t^{R}(M, N)$, defined as follows:

$$
\begin{aligned}
& e_{R}(M, N)=\sup \left\{n \in \mathbb{N} \mid \operatorname{Ext}_{R}^{n}(M, N) \neq 0\right\}, \\
& t^{R}(M, N)=\sup \left\{n \in \mathbb{N} \mid \operatorname{Tor}_{n}^{R}(M, N) \neq 0\right\} .
\end{aligned}
$$

A local ring $(Q, \mathfrak{n}, k)$ is called an (embedded) deformation of $R$ if $R \cong Q /(\boldsymbol{f})$, where $\boldsymbol{f}$ is a $Q$-regular sequence (contained in $\mathfrak{n}^{2}$ ). The ring $R$ is said to be a complete intersection if its $\mathfrak{m}$-adic completion $\widehat{R}$ has an embedded deformation which is a regular ring. Vanishing of (co)homology over complete intersections has remarkable properties, as proved by Avramov and Buchweitz [4, Theorem III]. They relate vanishing properties to homogeneous algebraic varieties $V_{R}^{*}(M) \subseteq \widetilde{k}^{c}$, defined in [3], where $c=\operatorname{codim} R$ and $\widetilde{k}$ denotes the algebraic closure of $k$.

Theorem AB. Let $R$ be a complete intersection local ring. One of the numbers $e_{R}(M, N), e_{R}(N, M), t^{R}(M, N)$ is finite if and only if all of them are less than or equal to $\operatorname{dim} R$, if and only if $\mathrm{V}_{R}^{*}(M) \cap \mathrm{V}_{R}^{*}(N)=0$.

An interesting consequence of Theorem $\mathrm{AB}$ is that the vanishing of $\operatorname{Ext}_{R}^{n}(M, N)$ for all $n \gg 0$ is symmetric in the two module variables. It is clear that a ring

Received by the editors November 6, 2001 and, in revised form, March 5, 2002.

2000 Mathematics Subject Classification. Primary 13D07, 13H10; Secondary 13D40.

Key words and phrases. Gorenstein rings, vanishing of Ext, CI-dimension.

(C)2002 American Mathematical Society 
with this property is Gorenstein. To study the symmetry in the vanishing of Ext, Huneke and Jorgensen [8] define a class of Gorenstein rings, called $A B$ rings, by the condition that $\sup \left\{e_{R}(M, N)\right\}$ is finite when the supremum is taken over all finite $R$-modules $M, N$ with $e_{R}(M, N)<\infty$. In [8, 3.1, 3.3(1), 4.1] they prove:

Theorem HJ. The ring $R$ is $A B$ if and only if every pair $(M, N)$ of finite $R$ modules with $e_{R}(M, N)<\infty$ (respectively $t^{R}(M, N)<\infty$ ) satisfies $e_{R}(M, N) \leq$ $\operatorname{dim} R$ and $e_{R}(N, M) \leq \operatorname{dim} R$ (respectively $t^{R}(M, N) \leq \operatorname{dim} R$ ).

Theorem AB shows that a complete intersection local ring is an $\mathrm{AB}$ ring. A noncomplete intersection example of $\mathrm{AB}$ rings is given by Gorenstein rings of minimal multiplicity and codimension at least 3: It is proved in 8, 3.5] that over such rings the finiteness of $e_{R}(M, N)$ or $t^{R}(M, N)$ implies one of the modules has finite projective dimension.

In Theorem 1 we identify another class of rings with this property.

Theorem 1. Let $R$ be a local Gorenstein ring such that $\operatorname{codim} R \leq 4$ and $\widehat{R}$ admits no embedded deformation. If one of the numbers $e_{R}(M, N), t^{R}(M, N)$ is finite, then $M$ or $N$ has finite projective dimension.

The theorem is proved in Section 2 as Theorem 2.3. The proof uses the fact that, for the rings in Theorem 1 , all finite $R$-modules have rational Poincaré series which share an explicitly known denominator: this is due to Jacobsson, Kustin, and Miller [13], 14], [15]. Preliminaries on rational Poincaré series are presented in Section 1, where we also give a new proof of the result on Gorenstein rings of minimal multiplicity. Clearly, such an approach is not applicable to rings that have finite modules with irrational Poincaré series. Such rings have been constructed by Bøgvad [6] in codimension 12 and higher.

To describe the aymptotic vanishing of cohomology over all Gorenstein rings with $\operatorname{codim} R \leq 4$, we use the notion of finite complete intersection dimension introduced by Avramov, Gasharov and Peeva [5]. An $R$-module $M$ is said to have finite $C I$ dimension if there exist a flat ring homomorphism $R \rightarrow R^{\prime}$ and a deformation $Q$ of $R^{\prime}$ such that $\operatorname{pd}_{Q}\left(M \otimes_{R} R^{\prime}\right)<\infty$. Note that any module of finite projective dimension has finite CI-dimension, and that if $R$ is a complete intersection, then every finite $R$-module has finite CI-dimension. The next statement is derived from the theorems presented above, as well as from expressions for $e_{R}(M, N)$ and $t^{R}(M, N)$ obtained by Araya, Yoshino, Avramov, and Buchweitz [1], 4]. It shows, in particular, that all Gorenstein rings of codimension at most 4 are AB:

Theorem 2. Let $R$ be a local Gorenstein ring with $\operatorname{codim} R \leq 4$.

If $e_{R}(M, N)<\infty$, then $M$ or $N$ has finite CI-dimension and

$$
\begin{aligned}
& e_{R}(M, N)=\operatorname{dim} R-\operatorname{depth}_{R} M, \\
& e_{R}(N, M)=\operatorname{dim} R-\operatorname{depth}_{R} N .
\end{aligned}
$$

If $t^{R}(M, N)<\infty$, then $M$ or $N$ has finite CI-dimension and

$$
t^{R}(M, N) \leq \max \left\{\operatorname{dim} R-\operatorname{depth}_{R} M, \operatorname{dim} R-\operatorname{depth}_{R} N\right\} .
$$

The theorem is proved as Theorem [3.4 in Section 3. An example is provided there to show that the conclusion regarding CI-dimension cannot be extended to high codimensions. However, it is not known (to the author) whether the other conclusions can be extended or not. 
Under the hypotheses of Theorem 1, asymptotic vanishing of Tor or Ext occurs only for trivial reasons: one of the modules has finite projective dimension. The situation is different if $R$ is a complete intersection with $\operatorname{codim} R \geq 2$. Over such rings, Avramov [3, 6.5] constructs for every linear subspace $H \subseteq \widetilde{k}^{c}$ an $R$-module $M$ of finite length with $\mathrm{V}_{R}^{*}(M)=H$; taking modules $M, N$ that correspond to nontrivial subspaces with zero intersection, one sees from Theorem AB that the numbers $e_{R}(M, N), e_{R}(N, M)$ and $t^{R}(M, N)$ are all finite.

We show that (co)homology may vanish for non-trivial reasons whenever $\widehat{R}$ has a non-regular embedded deformation:

Theorem 3. Let $R$ be a local Gorenstein ring with $\operatorname{codim} R \geq 2$. If $\widehat{R}$ has an embedded deformation, then there exist $R$-modules $M, N$ of finite length with $\operatorname{pd}_{R} M=\operatorname{pd}_{R} N=\infty$ and $e_{R}(M, N)=e_{R}(N, M)=t_{R}(M, N)=\operatorname{dim} R$.

This result is contained in the more general Corollary 4.4 proved in Section 4. The hypothesis on the codimension is necessary. Indeed, if $R$ is a Gorenstein ring with $\operatorname{codim} R \leq 1$, then $\widehat{R}=Q /(f)$ for a regular local $\operatorname{ring} Q$ and a $Q$-regular element $f$. For such rings, $t^{R}(M, N)<\infty$ or $e_{R}(M, N)<\infty$ implies that one of the modules $M$ or $N$ has finite projective dimension; cf. Huneke and Wiegand [9] 1.9], respectively Avramov and Buchweitz [4, 5.12].

\section{Rational Poincaré series}

In this section $(R, \mathfrak{m}, k)$ is a local ring and $M, N$ denote finite $R$-modules. We use several formal power series with integer coefficients associated to a finite $R$-module.

We let $\mathrm{P}_{M}^{R}(t)$ denote the Poincaré series of $M$ over $R$

$$
\mathrm{P}_{M}^{R}(t)=\sum_{i=0}^{\infty} \operatorname{rank}_{k} \operatorname{Tor}_{i}^{R}(M, k) t^{i} \in \mathbb{Z}[[t]]
$$

and $\mathrm{I}_{R}^{M}(M)$ denote the Bass series of $M$ over $R$

$$
\mathrm{I}_{R}^{M}(t)=\sum_{i=0}^{\infty} \operatorname{rank}_{k} \operatorname{Ext}_{R}^{i}(k, M) t^{i} \in \mathbb{Z}[[t]] .
$$

We also use the Hilbert series of $M$ over $R$, defined to be the series

$$
\mathrm{H}_{M}^{R}(t)=\sum_{i=0}^{\infty} \operatorname{rank}_{k}\left(\mathfrak{m}^{i} M / \mathfrak{m}^{i+1} M\right) t^{i} \in \mathbb{Z}[[t]] .
$$

Recall that $\mathrm{H}_{M}^{R}(t)$ represents a rational function with denominator $(1-t)^{\operatorname{dim} M}$.

1.1. Let $R \rightarrow R^{\prime}$ be a flat homomorphism of local rings such that $\mathfrak{m} R^{\prime}$ is the maximal ideal of $R^{\prime}$. For $M^{\prime}=M \otimes_{R} R^{\prime}$ and $N^{\prime}=N \otimes_{R} R^{\prime}$ there are equalities $\mathrm{P}_{M}^{R}(t)=\mathrm{P}_{M^{\prime}}^{R^{\prime}}(t)$ and $\mathrm{I}_{R}^{M}(t)=\mathrm{I}_{R^{\prime}}^{M^{\prime}}(t), e_{R}(M, N)=e_{R^{\prime}}\left(M^{\prime}, N^{\prime}\right)$ and $t^{R}(M, N)=$ $t^{R^{\prime}}\left(M^{\prime}, N^{\prime}\right), \operatorname{pd}_{R^{\prime}}\left(M^{\prime}\right)=\operatorname{pd}_{R} M$ and $\operatorname{id}_{R^{\prime}}\left(N^{\prime}\right)=\operatorname{id}_{R} N$. This situation applies in particular to the completion map $R \rightarrow \widehat{R}$.

1.2. Lemma. Assume there exists a polynomial $c(t)$ such that $c(t) \mathrm{P}_{M}^{R}(t) \in \mathbb{Z}[t]$ for each finite $R$-module $M$. If $c(t) \mathrm{P}_{k}^{R}(t)=(1+t)^{e} a(t)$ for an integer $e \geq 0$ and a polynomial $a(t) \in \mathbb{Z}[t]$ with $a(-1) \neq 0$, then $c(t)(1+t)^{m} \mathrm{I}_{R}^{N}(t) \in \mathbb{Z}[t]$ for each finite $R$-module $N$, where $m=\max \{0, \operatorname{dim} R-e\}$. 
Proof. Set $d=\operatorname{dim} R$. By Foxby [7, 3.10] there exists a finite $\widehat{R}$-module $L$ and a polynomial $g(t)$ with integer coefficients and degree less than $d$ such that

$$
\mathrm{I}_{R}^{N}(t)=g(t)+t^{d} \mathrm{P}_{L}^{\widehat{R}}(t) .
$$

As shown by Levin [16, p. 8] there exists an integer $n$ such that $M=L / \mathfrak{m}^{n} L$ satisfies

$$
\mathrm{P}_{L}^{\widehat{R}}(t)=\mathrm{P}_{M}^{\widehat{R}}(t)-t \mathrm{H}_{\mathfrak{m}^{n} L}^{\widehat{R}}(-t) \mathrm{P}_{k}^{\widehat{R}}(t) .
$$

The $\widehat{R}$-module $M$ has finite length, so considered as an $R$-module it has finite length and is complete. From subsection 1.1 we get $\mathrm{P}_{M}^{R}(t)=\mathrm{P}_{M}^{\widehat{R}}(t)$ and $\mathrm{P}_{k}^{R}(t)=$ $\mathrm{P}_{k}^{\widehat{R}}(t)$. Since $\operatorname{dim}_{\widehat{R}}\left(\mathfrak{m}^{n} L\right) \leq d$, we have $(1-t)^{d} \mathrm{H}_{\mathfrak{m}^{n} L}^{\widehat{R}}(t) \in \mathbb{Z}[t]$ and the conclusion follows.

The next result connects vanishing of (co)homology to Bass series and Poincaré series. Part (2) is due to C. Miller (cf. the proof of [17, 1.1]), part (1) is [4, 1.5(1)].

1.3. For finite $R$-modules $M, N$ the following hold:

(1) If $e_{R}(M, N)=0$, then $\mathrm{I}_{R}^{\mathrm{Hom}_{R}(M, N)}(t)=\mathrm{P}_{M}^{R}(t) \mathrm{I}_{R}^{N}(t)$.

(2) If $t^{R}(M, N)=0$, then $\mathrm{P}_{M \otimes_{R} N}^{R}(t)=\mathrm{P}_{M}^{R}(t) \mathrm{P}_{N}^{R}(t)$.

1.4. Definition. A factorization $c(t)=p(t) \cdot q(t) \cdot r(t)$ in $\mathbb{Z}[t]$ is said to be $\operatorname{good}$ if $p(t)=1$ or $p(t)$ is irreducible, $q(t)$ has non-negative coefficients, and $r(t)=1$ or $r(t)$ is irreducible and has no positive real root among its complex roots of minimal absolute value.

1.5. Proposition. Let $R$ be a local ring for which there exists a polynomial $c(t)$ such that $c(t) \mathrm{P}_{L}^{R}(t) \in \mathbb{Z}[t]$ for each finite $R$-module $L$. When $c(t)$ has a good factorization the following hold for all finite $R$-modules $M, N$ :

(1) If $e_{R}(M, N)<\infty$, then $\operatorname{pd}_{R} M<\infty$ or $\operatorname{id}_{R} N<\infty$.

(2) If $t^{R}(M, N)<\infty$, then $\operatorname{pd}_{R} M<\infty$ or $\operatorname{pd}_{R} N<\infty$.

Proof. We only give a proof of (1); the proof of (2) is similar.

Replacing $M$ by a syzygy, if necessary, we may assume $e_{R}(M, N)=0$. Set $b(t)=(1+t)^{d} c(t)$, where $d=\operatorname{dim} R$. By hypothesis and Lemma 1.2 we have

$$
b(t) \mathrm{P}_{M}^{R}(t)=m(t) \in \mathbb{Z}[t] \quad \text { and } \quad b(t) \mathrm{I}_{R}^{N}(t)=n(t) \in \mathbb{Z}[t] .
$$

Since $(1+t)^{d}$ is in $\mathbb{N}[t]$ it follows that $b(t)$ has a good factorization $b(t)=p(t) \cdot q(t)$. $r(t)$ as in Definition 1.4. Using section 1.3(1) we obtain

$$
b(t) \mathrm{I}_{R}^{\mathrm{Hom}_{R}(M, N)}(t)=b(t) \mathrm{P}_{M}^{R}(t) \mathrm{I}_{R}^{N}(t)=\frac{m(t) n(t)}{p(t) q(t) r(t)} .
$$

By Lemma 1.2 the expression on the left is a polynomial, so $p(t)$ divides $m(t) n(t)$. Since $p(t)=1$ or $p(t)$ is irreducible, two cases arise.

Case 1. $m(t)=p(t) m_{1}(t)$ with $m_{1}(t) \in \mathbb{Z}[t]$. We then have an equality

$$
q(t) \mathrm{P}_{M}^{R}(t)=\frac{m_{1}(t)}{r(t)} .
$$


Assume $r(t)$ does not divide $m_{1}(t)$. This means that the radius of convergence $\rho$ of these power series is finite. The series $q(t) \mathrm{P}_{M}^{R}(t)$ has non-negative coefficients, so by the Pringsheim Principle (cf. [22, 7.2]) $\rho$ is a singular point for it. On the other hand, the hypothesis on $r(t)$ implies that $\rho$ is not a singular point for $m_{1}(t) / r(t)$. This contradiction shows that $r(t)$ divides $m_{1}(t)$, so $q(t) \mathrm{P}_{M}^{R}(t)$ is a polynomial. As both factors have non-negative coefficients, we conclude that $\mathrm{P}_{M}^{R}(t)$ is a polynomial, that is, $\operatorname{pd}_{R}(M)<\infty$.

Case 2. $n(t)=p(t) n_{1}(t)$ with $n_{1}(t) \in \mathbb{Z}[t]$. An argument similar to the one above shows that $\mathrm{I}_{R}^{N}(t)$ is a polynomial, hence $\operatorname{id}_{R} N<\infty$.

The two parts of the proposition can be combined when the ring is Gorenstein:

1.6. If $R$ is Gorenstein, then $\operatorname{id}_{R} N<\infty$ is equivalent to $\operatorname{pd}_{R} N<\infty$.

Let $R$ be a Gorenstein ring with $\operatorname{codim} R \geq 2$. It is known that $R$ has multiplicity at least codim $R+2$; cf. [19, 3.2]. When equality holds, $R$ is said to be Gorenstein of minimal multiplicity.

1.7. Remark. If $R$ is Gorenstein of minimal multiplicity with $\operatorname{codim} R \geq 2$, then $c(t) \mathrm{P}_{M}^{R}(t) \in \mathbb{Z}[t]$ for each finite $R$-module $M$, where $c(t)=1-(\operatorname{codim} R) t+t^{2}$.

To see this, we may assume that $k$ is infinite: if not, then $R^{\prime}=R[t]_{\mathfrak{m}[t]}$ has mult $R^{\prime}=$ mult $R$ and we apply section [1.1. It also suffices to consider maximal Cohen-Macaulay modules $M$. If $\operatorname{dim} R=0$, then $\mathfrak{m}^{3}=0$ and the result is proved by $\mathrm{Sjödin}$ [20]. If $\operatorname{dim} R>0$, then there exists a maximal $R$-regular sequence $\boldsymbol{g}$ such that $\operatorname{codim} R=\operatorname{codim} R /(\boldsymbol{g})$ and mult $R=\operatorname{mult} R /(\boldsymbol{g})$. Since $M$ is maximal Cohen-Macaulay, this sequence is also $M$-regular. The isomorphisms $\operatorname{Tor}_{n}^{R}(M, k) \cong$ $\operatorname{Tor}_{n}^{R /(\boldsymbol{g})}(M / \boldsymbol{g} M, k)$ then yield $\mathrm{P}_{M}^{R}(t)=\mathrm{P}_{M / \boldsymbol{g} M}^{R /(\boldsymbol{g})}(t)$. The conclusion follows by the dimension 0 case.

If $\operatorname{codim} R>2$, then the polynomial $c(t)=1-(\operatorname{codim} R) t+t^{2}$ is irreducible, hence $c(t)=c(t) \cdot 1 \cdot 1$ is a good factorization. From Proposition 1.5 and Remark 1.7, we obtain a new proof of [8, 3.5]:

1.8. Corollary. Let $R$ be a local Gorenstein ring of minimal multiplicity with $\operatorname{codim} R \geq 3$. If $M, N$ are finite $R$-modules such that one of the numbers $e_{R}(M, N)$, $t^{R}(M, N)$ is finite, then $M$ or $N$ has finite projective dimension.

\section{Gorenstein RINGS OF SMALL CODIMENSION}

In this section $R$ denotes a Gorenstein ring of codimension at most 4 which is not a complete intersection. Over such rings, the Poincaré series of all finite $R$-modules are rational and share a common denominator. This result, and the form of the denominator, were obtained by Jacobsson [13] in codimension 3, by Jacobsson, Kustin and Miller [14, 2.3] in codimension 4, characteristic different from 2 and by Kustin [15] in codimension 4 and characteristic different from 3. We collect below the relevant information; we refer to [2, §3] for details. 
2.1. There exists a polynomial $c(t) \in \mathbb{Z}[t]$ such that $c(t) \mathrm{P}_{M}^{R}(t) \in \mathbb{Z}[t]$ for all finite $R$ modules $M$. This polynomial has the form $c(t)=d(t)(1+t)^{m}$, where the polynomial $d(t) \in \mathbb{Z}[t]$ and the non-negative integer $m$ are as follows:

\begin{tabular}{|c|c|c|c|c|}
\hline type & $\operatorname{codim} R$ & $d(t)$ & $m$ & restrictions \\
\hline $\mathbf{G}(l+1)$ & 3 & $1-t-l t^{2}-t^{3}+t^{4}$ & 1 & $l \geq 4$ \\
\hline $\mathbf{G T E}$ & 4 & $1-2 t-(l-2) t^{2}+t^{3}+t^{4}-t^{5}$ & 2 & $l \geq 5$ \\
\hline $\mathbf{G G O}$ & 4 & $1-2 t-(l-2) t^{2}-2 t^{3}+t^{4}$ & 2 & $l \geq 5$ \\
\hline $\mathbf{G H}(p)$ & 4 & $1-2 t-(l-2) t^{2}+(p-2) t^{3}+2 t^{4}-t^{5}$ & 2 & $1 \leq p \leq l \geq 5$ \\
\hline
\end{tabular}

Avramov [2 3.1] determines when $\widehat{R}$ has an embedded deformation:

2.2. The ring $\widehat{R}$ admits an embedded deformation if and only if $d(1)=0$, if and only if $R$ is of type $\mathbf{G H}(p)$, with $p=l$.

The proof of the next result goes through a careful examination of the polynomials in the chart above. Earlier, Sun [21] used a case-by-case analysis of these polynomials to prove that the Betti numbers of finite modules over a Gorenstein ring of codimension at most 4 are eventually non-decreasing.

2.3. Theorem. Let $R$ be a local Gorenstein ring such that $\operatorname{codim} R \leq 4$ and $\widehat{R}$ admits no embedded deformation. If $M, N$ are finite $R$-modules and one of the numbers $e_{R}(M, N), t^{R}(M, N)$ is finite, then $M$ or $N$ has finite projective dimension.

Proof. By Proposition 1.5 and section 1.6 it suffices to show that the common denominator $c(t)$ has a good factorization. Since $(1+t)^{m}$ has non-negative coefficients, this will follow once we prove that $d(t)$ has a good factorization.

If $d(t)$ is irreducible, then $d(t)=d(t) \cdot 1 \cdot 1$ is a good factorization. For the rest of the proof we assume that $d(t)$ is reducible. If $d(t)$ has a linear factor, then $d(-1)=0$. Indeed, the only possible rational roots of $d(t)$ are \pm 1 , and $d(1)=0$ is excluded by section 2.2. For each type of polynomial in the chart, we study the factorization of $d(t)$ in the two remaining cases: $d(-1)=0$ and $d(t)$ has no linear factor.

$\mathbf{G}(l+1)$ or GGO: If $d(-1)=0$, then $l=4$ or $l=8$. Thus, we have good factorizations $d(t)=\left(1-3 t+t^{2}\right) \cdot(1+t)^{2} \cdot 1$ or $d(t)=\left(1-4 t+t^{2}\right) \cdot(1+t)^{2} \cdot 1$.

If $d(t)$ has no linear factor, then $d(t)=\left(1+a t+\varepsilon t^{2}\right)\left(1+b t+\varepsilon t^{2}\right)$ with $a, b \in \mathbb{Z}$, both factors irreducible, and $\varepsilon= \pm 1$. If $\varepsilon=-1$, then comparison of the coefficients of $t$ and $t^{3}$ gives $a+b<0$ and $-a-b<0$, so this case does not occur. If $\varepsilon=1$, then comparison of the coefficients of $t^{2}$ gives $a b<0$, hence we may assume $b>0$, and then $d(t)=\left(1+a t+t^{2}\right) \cdot\left(1+b t+t^{2}\right) \cdot 1$ is a good factorization.

GTE: If $d(-1)=0$, then $l=6$ and $d(t)=\left(1-4 t+3 t^{2}-t^{3}\right) \cdot(1+t)^{2} \cdot 1$ is a good factorization.

If $d(t)$ has no linear factor, then $d(t)=\left(1+a t+\varepsilon t^{2}\right)\left(1+b t+c t^{2}-\varepsilon t^{3}\right)$ with $a, b, c \in \mathbb{Z}$, both factors irreducible, and $\varepsilon= \pm 1$. Comparing coefficients, we get:

$$
\begin{aligned}
a+b & =-2, \\
a c+\varepsilon b-\varepsilon & =1, \\
\varepsilon c-\varepsilon a & =1 .
\end{aligned}
$$


Using the first and the last equality to eliminate $b$ and $c$ from the middle, we obtain $a^{2}=1+3 \varepsilon$. If $\varepsilon=-1$, then $a^{2}=-2$, which is not possible. If $\varepsilon=1$, then $a^{2}=4$, hence $a= \pm 2$, and this contradicts the assumption that $1+a t+\varepsilon t^{2}$ is irreducible.

$\mathbf{G H}(p)$ : If $d(-1)=0$, then $p+l=10$ and $d(t)=e(t)(1+t)$, where $e(t)=$ $1-3 t+(p-5) t^{2}+3 t^{3}-t^{4}$. Since $1+t$ has non-negative coefficients, the polynomial $d(t)$ has a good factorization if and only if $e(t)$ has a good factorization. If $e(t)$ is irreducible, then $e(t)=e(t) \cdot 1 \cdot 1$ is a good factorization, so we assume $e(t)$ is reducible. If $e(t)$ has a linear factor, then $e(-1)=0$, hence $p=5$. It follows that $p=l=5$, and this is ruled out by section [2.2. If $e(t)$ has no linear factor, then $e(t)=\left(1+a t-t^{2}\right)\left(1+b t+t^{2}\right)$ with both factors irreducible and $a, b \in \mathbb{Z}$. Comparing the coefficients of $t$ and $t^{3}$, we get $a+b=-3$ and $a-b=3$, hence $a=0$. This contradicts the hypothesis that $1+a t-t^{2}$ is irreducible.

If $d(t)$ has no linear factor, then $d(t)=\left(1+a t+\varepsilon t^{2}\right)\left(1+b t+c t^{2}-\varepsilon t^{3}\right)$ with $a, b, c \in \mathbb{Z}$, both factors irreducible, and $\varepsilon= \pm 1$. Comparison of coefficients yields

$$
\begin{aligned}
a+b & =-2, \\
a b+c+\varepsilon & =-l+2, \\
a c+\varepsilon b-\varepsilon & =p-2, \\
\varepsilon c-\varepsilon a & =2 .
\end{aligned}
$$

These equalities yield $-a^{2}-a=-l+2-3 \varepsilon$ and $a^{2}+\varepsilon a=p-2+3 \varepsilon$, so $\varepsilon a-a=p-l$. By sections 2.1 and 2.2 we have $p<l$, hence $\varepsilon=-1$ and $2 a=l-p>0$. Thus, $d(t)=\left(1+b t+c t^{2}+t^{3}\right) \cdot 1 \cdot\left(1+a t-t^{2}\right)$ is a good factorization.

\section{Finite CI-Dimension}

In this section we let $R$ denote a local ring and let $M, N$ be finite $R$-modules.

3.1. We refer to the Introduction for the definition of finite CI-dimension and we recall below the basic examples:

3.1.1. If $R$ is a complete intersection, then $M$ has finite CI-dimension.

3.1.2. If $\operatorname{pd}_{R} M<\infty$, then $M$ has finite CI-dimension.

3.2. When one of the modules $M$ or $N$ has finite CI-dimension, several (in)equalities involving the numbers $e_{R}(M, N)$ and $t^{R}(M, N)$ are known.

The inequality below follows from [4, 4.9] and [5, 1.4]:

3.2.1. If $t^{R}(M, N)<\infty$ and $M$ has finite CI-dimension, then

$$
t^{R}(M, N) \leq \operatorname{depth} R-\operatorname{depth}_{R} M .
$$

A formula for $e_{R}(M, N)$ is given by Araya and Yoshino [1, 4.2]:

3.2.2. If $e_{R}(M, N)<\infty$ and $M$ has finite CI-dimension, then

$$
e_{R}(M, N)=\operatorname{depth} R-\operatorname{depth}_{R} M .
$$

Over Gorenstein rings the equality of 3.2 .2 is more generally valid:

3.3. Lemma. If $R$ is Gorenstein, $e_{R}(M, N)$ is finite and $N$ has finite CI-dimension, then

$$
e_{R}(M, N)=\operatorname{depth} R-\operatorname{depth}_{R} M .
$$


Proof. If $\operatorname{pd}_{R} N<\infty$, then $\operatorname{id}_{R} N<\infty$ (see section [1.6), so the equality is given by a result of Ischebeck [10, 2.6]. In general, there exists a flat ring homomorphism $R \rightarrow R^{\prime}$ and a deformation $Q$ of $R^{\prime}$ such that $\operatorname{pd}_{Q}\left(M \otimes_{R} R^{\prime}\right)<\infty$. By section 1.1we may assume that $R=R^{\prime}$ and then $e_{Q}(M, N)=\operatorname{depth} Q-\operatorname{depth}_{Q} M$. A standard argument (cf. 1, 2.6]) then gives

$$
e_{R}(M, N)=e_{Q}(M, N)-\left(\operatorname{depth}_{Q} R-\operatorname{depth} Q\right)=\operatorname{depth} R-\operatorname{depth}_{R} M .
$$

The next theorem is the main result of this section. It shows, in particular, that every Gorenstein ring of codimension at most 4 is $\mathrm{AB}$.

3.4. Theorem. Let $R$ be a local Gorenstein ring with $\operatorname{codim} R \leq 4$ and let $M, N$ be finite $R$-modules.

If $e_{R}(M, N)$ is finite, then $M$ or $N$ has finite CI-dimension and

$$
\begin{aligned}
& e_{R}(M, N)=\operatorname{dim} R-\operatorname{depth}_{R} M, \\
& e_{R}(N, M)=\operatorname{dim} R-\operatorname{depth}_{R} N .
\end{aligned}
$$

If $t^{R}(M, N)$ is finite, then $M$ or $N$ has finite CI-dimension and

$$
t^{R}(M, N) \leq \max \left\{\operatorname{dim} R-\operatorname{depth}_{R} M, \operatorname{dim} R-\operatorname{depth}_{R} N\right\} .
$$

Proof. In view of the results recalled in section 3.2 Lemma 3.3, and Theorem HJ stated in the Introduction, it suffices to prove the following claim: If $e_{R}(M, N)$ or $t^{R}(M, N)$ is finite, then one of the modules $M, N$ has finite CI-dimension.

By 3.1.1, the claim holds when $R$ is a complete intersection. If $R$ has no embedded deformation, then the statement results from Theorem 2.3, in view of 3.1 .2

It thus remains to treat the case when $R$ is not a complete intersection and has an embedded deformation $Q$. This only happens when $\operatorname{codim} R=4$ and $Q$ has no embedded deformation (otherwise, $\widehat{R}$ deforms to a Gorenstein ring $Q^{\prime}$ with $\operatorname{codim} Q^{\prime} \leq 2$, and such a ring is a complete intersection). Standard arguments (see [1, 2.6] for example) show that $e_{R}(M, N)<\infty$ implies $e_{Q}(\widehat{M}, \widehat{N})<\infty$ and $t^{R}(M, N)<\infty$ implies $t^{Q}(\widehat{M}, \widehat{N})<\infty$. By Theorem $1, \widehat{M}$ or $\widehat{N}$ then has finite projective dimension over $Q$, and thus the corresponding module has finite CIdimension over $R$.

We note some further applications of the conclusion on finite CI-dimension of Theorem 3.4.

Araya and Yoshino 1, 4.2] give a self-test for finite projective dimension when the CI-dimension is finite: If $M$ has finite CI-dimension, then there is an equality $e_{R}(M, M)=\operatorname{pd}_{R}(M)$. In view of Theorem 3.4 we have

3.5. Corollary. There is an equality $e_{R}(M, M)=\operatorname{pd}_{R}(M)$.

When $M$ or $N$ has finite CI-dimension, (1) below is proved by Jorgensen [13. 2.2] and (2) is proved by Araya and Yoshino [1, 2.5] (see also Iyengar [11, 4.3] for the case $q=0$ ) over any local ring $R$. By Theorem 3.4 we have

3.6. Corollary. Set $d_{R}(M, N)=\operatorname{depth} R-\operatorname{depth}_{R} M-\operatorname{depth}_{R} N$.

If $t^{R}(M, N)=q<\infty$, then the following hold:

(1) $t^{R}(M, N)=\sup \left\{d_{R_{\mathfrak{p}}}\left(M_{\mathfrak{p}}, N_{\mathfrak{p}}\right) \mid \mathfrak{p} \in \operatorname{Supp} M \cap \operatorname{Supp} N\right\}$.

(2) If $q=0$ or $\operatorname{depth}_{R} \operatorname{Tor}_{q}^{R}(M, N) \leq 1$, then

$$
t^{R}(M, N)=d_{R}(M, N)+\operatorname{depth}_{R} \operatorname{Tor}_{q}^{R}(M, N) .
$$


The conclusion on CI-dimension of Theorem 3.4 does not extend to higher codimensions: There exist rings of any codimension greater than or equal to 6 and $R$-modules $M, N$ of infinite CI-dimension such that $e_{R}(M, N)<\infty$. The example below is based on a construction in [8, 4.3].

3.7. Example. Let $(S, \mathfrak{s}, k)$ and $(T, \mathfrak{t}, k)$ be two Gorenstein rings that are essentially of finite type over a field $k$ where none of them is a complete intersection, so they have codimension at least 3 . Set $\mathfrak{p}=\mathfrak{s} \otimes_{k} T+S \otimes_{k} \mathfrak{t}$. The local ring $R=\left(S \otimes_{k} T\right)_{\mathfrak{p}}$ is then Gorenstein and has $\operatorname{codim} R=\operatorname{codim} T+\operatorname{codim} S \geq 6$. The $R$-modules $M=S \otimes_{k} k$ and $N=k \otimes_{k} T$ satisfy $e_{R}(M, N) \leq \operatorname{dim} R$ by [8, 4.3]. Since $S$ is not a complete intersection, $k$ has infinite CI-dimension over it; cf. [5, 1.3]. The ring $S$ is faithfully flat as a $k$-module, so [5] 1.13] yields CI-dim $R \geq \geq \mathrm{CI}-\operatorname{dim}_{S} k=\infty$. By symmetry, $N$ also has infinite CI-dimension over $R$.

\section{EMBEDDED DEFORMATIONS}

In this section we construct examples to show that vanishing of (co)homology can occur for non-trivial reasons.

Throughout the section, $S$ denotes a local commutative noetherian ring. Our examples are based on the existence of modules with periodic resolutions over rings with embedded deformations. We recall the relevant definitions and results:

Let $a \geq 1$ be an integer. The minimal free resolution of a finite $S$-module $U$ is said to be periodic of period $a$ if $\operatorname{Syz}_{n}^{S}(U) \cong \operatorname{Syz}_{n+a}^{S}(U)$ for all $n \geq 0$. The following result is due to Avramov, Gasharov and Peeva [5, 3.2]:

4.1. If $S \cong Q /(f)$ for a non-regular local $\operatorname{ring}(Q, \mathfrak{n})$ and a $Q$-regular element $f \in \mathfrak{n}^{2}$, then there exists a finite $S$-module $U$ whose minimal $S$-free resolution is periodic of period 2 and $\operatorname{pd}_{Q} U=1$. In particular, $\operatorname{pd}_{S} U=\infty$ and $\operatorname{depth}_{S} U=\operatorname{depth} S$.

4.2. Theorem. If $S \cong Q /(f)$ for a non-regular local ring $(Q, \mathfrak{n})$ and a $Q$-regular element $f \in \mathfrak{n}^{2}$, and $U$ is the $S$-module from section 4.1 , then there exists a finite $S$-module $V$ with $\operatorname{depth}_{S} V=\operatorname{depth} S$ such that

$$
\operatorname{id}_{S} V=\infty=\operatorname{pd}_{S} V \quad \text { and } \quad e_{S}(U, V)=0=t_{S}(U, V) .
$$

If, furthermore, $S$ is Gorenstein, then $e_{S}(V, U)=0$ as well.

Proof. Since $Q$ is not regular, we have $\operatorname{pd}_{Q} k=\infty$. Set $s=\operatorname{depth} Q+2$. By 18 . $\S 2]$ we have $\operatorname{depth}_{Q} \operatorname{Syz}_{i}^{Q}(k)=\operatorname{depth} Q$ for each $i \geq s$. If $Q$ is Gorenstein, then $\operatorname{id}_{Q} \operatorname{Syz}_{i}^{Q}(k)=\infty$ for each $i \geq 0$ by section 1.6 and we set $V^{\prime}=\operatorname{Syz}_{s}^{Q}(k)$. Otherwise, one of the syzygy modules in the short exact sequence $0 \rightarrow \operatorname{Syz}_{s+1}^{Q}(k) \rightarrow Q^{b} \rightarrow$ $\operatorname{Syz}_{s}^{Q}(k) \rightarrow 0$ has infinite injective dimension and we let $V^{\prime}$ be this module.

Set $V=V^{\prime} / f V^{\prime}$. Note that $f$ is a $V^{\prime}$-regular element and $\operatorname{depth}_{S} V=\operatorname{depth} S$. The isomorphisms $\operatorname{Ext}_{S}^{n}(k, V) \cong \operatorname{Ext}_{Q}^{n+1}\left(k, V^{\prime}\right)$ and $\operatorname{Tor}_{n}^{S}(k, V) \cong \operatorname{Tor}_{n}^{Q}\left(k, V^{\prime}\right)$ show that $\operatorname{id}_{S} V=\operatorname{pd}_{S} V=\infty$. The isomorphisms $\operatorname{Ext}_{S}^{n}(U, V) \cong \operatorname{Ext}_{Q}^{n+1}\left(U, V^{\prime}\right)$ and $\operatorname{Tor}_{n}^{S}(U, V) \cong \operatorname{Tor}_{n}^{Q}\left(U, V^{\prime}\right)$ yield $e_{S}(U, V)=0$ and $t^{S}(U, V) \leq 1$, because $\operatorname{pd}_{Q} U=1$. The periodicity of the minimal free resolution of $U$ gives $\operatorname{Tor}_{n}^{S}(U, V) \cong$ $\operatorname{Tor}_{n+2}^{S}(U, V)$ for all $n>0$, hence $t^{S}(U, V)=0$.

If $S$ is a Gorenstein ring, then $Q$ is Gorenstein, hence $\operatorname{id}_{Q} U<\infty$ by section 1.6 . The isomorphisms $\operatorname{Ext}_{S}^{n}(V, U) \cong \operatorname{Ext}_{Q}^{n}\left(V^{\prime}, U\right)$ then show that $e_{S}(V, U)<\infty$. Since the $S$-module $U$ has finite CI-dimension, Lemma 3.3 yields $e_{S}(V, U)=0$. 
The next lemma is an extension of the fact that if $U$ is a finite $S$-module and $\bar{U}=U / g U$ for a $U$-regular element $g$, then $\operatorname{pd}_{S} \bar{U}=\operatorname{pd}_{S} U+1$ and $\operatorname{id}_{S} \bar{U}=\operatorname{id}_{S} U$.

4.3. Lemma. Let $S$ be a local ring, $U, V$ be finite $S$-modules and $g \in S$ a $U$-regular element. For the $S$-module $\bar{U}=U / g U$ the following hold:

(1) $e_{S}(\bar{U}, V)=e_{S}(U, V)+1$.

(2) $e_{S}(V, \bar{U})=e_{S}(V, U)$.

(3) $t^{S}(U, V) \leq t^{S}(\bar{U}, V) \leq t^{S}(U, V)+1$.

If $g V=0$ or $V$ has finite length, then $t^{S}(\bar{U}, V)=t^{S}(U, V)+1$.

(4) If the minimal free resolution of $\operatorname{Syz}_{s}^{S}(U)$ is periodic of period 2 for an integer $s \geq 0$, then the minimal free resolution of $\operatorname{Syz}_{s+1}^{S}(\bar{U})$ is periodic of period 2.

Proof. Properties (1) to (3) are deduced using the long exact sequences induced by the exact sequence $0 \rightarrow U \stackrel{g}{\rightarrow} U \rightarrow \bar{U} \rightarrow 0$.

We only give the proof of (3). In this case, the long exact sequence is

$$
\begin{aligned}
\ldots \rightarrow \operatorname{Tor}_{n+1}^{S}(\bar{U}, V) & \rightarrow \operatorname{Tor}_{n}^{S}(U, V) \stackrel{g}{\rightarrow} \operatorname{Tor}_{n}^{S}(U, V) \rightarrow \\
& \rightarrow \operatorname{Tor}_{n}^{S}(\bar{U}, V) \rightarrow \operatorname{Tor}_{n-1}^{S}(U, V) \rightarrow \ldots
\end{aligned}
$$

Nakayama's Lemma shows that if $\operatorname{Tor}_{n}^{S}(U, V) \neq 0$, then $\operatorname{Tor}_{n}^{S}(\bar{U}, V) \neq 0$, hence $t^{S}(U, V) \leq t^{S}(\bar{U}, V)$. To prove the remaining statements it suffices to assume $t^{S}(U, V)=p<\infty$. The long exact sequence shows that if $\operatorname{Tor}_{i}^{S}(U, V)=0$ for $i=n$ and $i=n-1$, then $\operatorname{Tor}_{n}^{S}(\bar{U}, V)=0$. We conclude $t^{S}(\bar{U}, V) \leq p+1$. If $g V=0$ or $V$ has finite length, then multiplication by $g$ on $\operatorname{Tor}_{p}^{S}(U, V)$ has non-zero kernel, hence $\operatorname{Tor}_{p+1}^{S}(\bar{U}, V) \neq 0$ and thus $t^{S}(\bar{U}, V)=p+1$.

(4) Let $F$ be a minimal free resolution of $U$. The mapping cone of the homomorphism $F \stackrel{g}{\rightarrow} F$ is a minimal free resolution of $\bar{U}$, hence we have $\operatorname{Syz}_{n}^{S}(\bar{U}) \cong$ $\operatorname{Syz}_{n}^{S}(U) / g \operatorname{Syz}_{n}^{S}(U) \oplus \operatorname{Syz}_{n-1}^{S}(U)$ for all $n \geq 0$, and the conclusion follows.

4.4. Corollary. Let $R$ be a d-dimensional Cohen-Macaulay local ring such that $\operatorname{codim} R \geq 2$ and $\widehat{R} \cong Q /(f)$ for a local ring $(Q, \mathfrak{n})$ and a $Q$-regular element $f \in \mathfrak{n}^{2}$.

There exist $R$-modules $M, N$ of finite length such that $\operatorname{pd}_{Q} M=d+1$, the minimal $R$-free resolution of $\operatorname{Syz}_{d}^{R}(M)$ is periodic of period 2 and

$$
\operatorname{id}_{R} N=\infty=\operatorname{pd}_{R} N \quad \text { and } \quad e_{R}(M, N)=d=t^{R}(M, N) .
$$

If, furthermore, $R$ is Gorenstein, then $e_{R}(N, M)=d$.

Proof. Since $\operatorname{codim} R \geq 2$, the $\operatorname{ring} Q$ is not regular. Set $S=\widehat{R}$ and let $U$ and $V$ be as in Theorem 4.2. Choose a $U$ - and $V$-regular sequence $\boldsymbol{g}$ of length $d$ and set $M=U / \boldsymbol{g} U$ and $N=V / \boldsymbol{g} V$. By Lemma 4.3 we have $\operatorname{id}_{S} N=\infty=\operatorname{pd}_{S} N$ and the minimal $S$-free resolution of $\operatorname{Syz}_{d}^{S}(M)$ is periodic of period 2. Using Theorem 4.2 and Lemma 4.3 we conclude $e_{S}(M, N)=e_{S}(U, V)+d=d$ and $t^{S}(M, N)=$ $t^{S}(U, N)+d<\infty$. Since the minimal free resolution of $U$ is periodic of period 2, we have $\operatorname{Tor}_{n}^{S}(U, N) \cong \operatorname{Tor}_{n+2}^{S}(U, N)$ for all $n>0$, hence $t^{S}(U, N)=0$ and thus $t^{S}(M, N)=d$. If $S$ is a Gorenstein ring, then $e_{S}(N, M)=e_{S}(V, U)+d=d$ by Theorem 4.2 and Lemma 4.3 .

It remains to note that $M$ and $N$ are $\widehat{R}$-modules of finite length, hence, considered as $R$-modules, they have finite length and are complete. The desired conclusion now follows by applying section 1.1 


\section{ACKNOWLEDGMENT}

I thank my thesis advisor Luchezar Avramov for inspiring the development of this paper and for his constant help.

\section{REFERENCES}

[1] T. Araya, Y. Yoshino, Remarks on a depth formula, a grade inequality, and a conjecture of Auslander, Comm. Algebra 26 (1998), 3793-3806. MR 99h:13010

[2] L. L. Avramov, Homological asymptotics of modules over local rings, Commutative Algebra (Berkeley, 1987), MSRI Publ. 15, Springer, New York 1989; pp. 33-62. MR 90i:13014

[3] L. L. Avramov, Modules of finite virtual projective dimension, Invent. Math. 96 (1989), 71-101. MR 90g:13027

[4] L. L. Avramov, R.-O. Buchweitz, Support varieties and cohomology over complete intersections, Invent. Math. 142 (2000), 285-318. MR 2001j:13017

[5] L. L. Avramov, V. N. Gasharov, I. V. Peeva, Complete intersection dimension, Publ. Math. I.H.E.S. 86 (1997), 67-114. MR 99c:13033

[6] B. Bøgvad, Gorenstein rings with transcendental Poincaré series, Math. Scand. 53 (1983), 5-15. MR 85c:13012

[7] H.-B. Foxby, On the $\mu^{i}$ in a minimal injective resolution. II, Math. Scand. 41 (1977), 19-44. MR 57:16355

[8] C. Huneke, D. Jorgensen, Symmetry in the vanishing of Ext over Gorenstein rings, Preprint, 2001.

[9] C. Huneke, R. Wiegand, Tensor products of modules, rigidity, and local cohomology, Math. Scand. 81 (1997), 161-183. MR 2000d:13027

[10] F. Ischebeck, Eine Dualität zwischen den Funktoren Ext und Tor, J. Algebra 11 (1969), 510-531. MR 38:5894

[11] S. Iyengar, Depth for complexes, and intersection theorems, Math. Z. 230 (1999), 545-567. MR 2000a:13027

[12] D. Jorgensen, A generalization of the Auslander-Buchsbaum formula, J. Pure Appl. Algebra 144 (1999), 145-155. MR 2000k:13010

[13] C. Jacobsson, On the positivity of the deviations of a local ring, Uppsala Univ., Dept. of Math., Report 1983:2.

[14] C. Jacobsson, A. R. Kustin, M. Miller, The Poincaré series of a codimension four Gorenstein ring is rational, J. Pure Appl. Algebra 38 (1985), 255-275. MR 87f:13021

[15] A. R. Kustin, Gorenstein algebras of codimension four and characteristic two, Communications in Algebra 15 (1987), 2417-2429. MR 88j:13020

[16] G. Levin, Poincaré series of modules over local rings, Proc. Amer. Math. Soc. 72 (1978), 6-10. MR 81b:13009

[17] C. Miller, Complexity of tensor products of modules and a theorem of Huneke-Wiegand, Proc. Amer. Math. Soc. 126 (1998), 53-60. MR 98c:13022

[18] S. Okiyama, A local ring is CM if and only if its residue field has a CM syzygy, Tokyo J. Math. 14 (1991), 489-500. MR 92m:13017

[19] J. D. Sally, Tangent cones at Gorenstein singularities, Compositio Math. 40 (1980), 167-175. MR 81e:14004

[20] G. Sjödin, The Poincaré series of modules over a local Gorenstein ring with $\mathfrak{m}^{3}=0$, Mathematiska Institutionen, Stockholms Universitet, Preprint 2, 1979.

[21] L.-C. Sun, Growth of Betti numbers of modules over local rings of small embedding dimension or small linkage number, J. Pure Appl. Algebra 96 (1994), 57-71. MR 95j:13014

[22] E. C. Titchmarsh, The theory of functions, Oxford University Press, London, 1939.

Department of Mathematics, Purdue University, West Lafayette, Indiana 47907

E-mail address: Imsega@math.purdue.edu

Current address: Mathematical Sciences Research Institute, 1000 Centennial Drive, Berkeley, California 94720

E-mail address: lsega@msri.org 